\title{
Targeting the kidney and glucose excretion with dapagliflozin: preclinical and clinical evidence for SGLT2 inhibition as a new option for treatment of type 2 diabetes mellitus
}

This article was published in the following Dove Press journal:

Diabetes, Metabolic Syndrome and Obesity: Targets and Therapy

18 July 2012

Number of times this article has been viewed

\author{
Jean MWhaley' \\ Mark Tirmenstein ${ }^{2}$ \\ Timothy P Reilly \\ Simon M Poucher ${ }^{3}$ \\ JoAnne Saye ${ }^{4}$ \\ Shamik Parikh ${ }^{5}$ \\ James F List ${ }^{6}$
}

'Bristol-Myers Squibb, Metabolic Disease Discovery Biology, Research and Development, Princeton, NJ, USA; ${ }^{2}$ Bristol-Myers Squibb, Drug Safety Evaluation, Research and Development, New Brunswick and Princeton, NJ, USA; ${ }^{3}$ AstraZeneca, Cardiovascular and Gastrointestinal Innovative Medicines Science Unit, Alderley Park, Macclesfield, Cheshire, UK; ${ }^{4}$ AstraZeneca, Global Safety Assessment, Research and Development, Wilmington, DE, USA; ${ }^{5}$ AstraZeneca, Cardiovascular, Clinical Development, Wilmington, DE, USA; 'Bristol-Myers Squibb, Global Clinical Development, Research and Development, Princeton, NJ, USA

Correspondence: Jean Whaley Bristol Myers-Squibb, 3 I I PenningtonRocky Hill Road, Mail Code 2I-2.0I, Pennington, NJ 08534, USA

$\mathrm{Tel}+\mathrm{l} 6098183380$

Fax + I 6098183600

Email jean.whaley@bms.com
Abstract: Sodium-glucose cotransporter-2 (SGLT2) inhibitors are a novel class of glucuretic, antihyperglycemic drugs that target the process of renal glucose reabsorption and induce glucuresis independently of insulin secretion or action. In patients with type 2 diabetes mellitus, SGLT2 inhibitors have been found to consistently reduce measures of hyperglycemia, including hemoglobin A1c, fasting plasma glucose, and postprandial glucose, throughout the continuum of disease. By inducing the renal excretion of glucose and its associated calories, SGLT2 inhibitors reduce weight and have the potential to be disease modifying by addressing the caloric excess that is believed to be one of the root causes of type 2 diabetes mellitus. Additional benefits, including the possibility for combination with insulin-dependent antihyperglycemic drugs, a low potential for hypoglycemia, and the ability to reduce blood pressure, were anticipated from the novel mechanism of action and have been demonstrated in clinical studies. Mechanism-related risks include an increased incidence of urinary tract and genital infections and the possibility of over-diuresis in volume-sensitive patients. Taken together, the results of Phase III clinical studies generally point to a positive benefit-risk ratio across the continuum of diabetes patients. To date, data on dapagliflozin, a selective SGLT2 inhibitor in development, demonstrate that the kidney is an efficacious and safe target for therapy, and that SGLT2 inhibition may have benefits for patients with type 2 diabetes mellitus beyond glycemic control.

Keywords: type 2 diabetes mellitus, SGLT2, dapagliflozin, mechanism of action

\section{Introduction}

Because uncontrolled hyperglycemia is a risk factor for diabetes complications (neuropathy, retinopathy, nephropathy) as well as cardiovascular disease, the primary goal of therapy for type 2 diabetes mellitus (T2DM) is glycemic control. Early, intensive control of plasma glucose has been shown to have long-term "legacy effects" for microvascular complications. It is also critically important to control a range of cardiovascular risk factors in these inherently at-risk patients. ${ }^{1-3}$ Weight is of particular concern because an estimated $79 \%-85 \%$ of patients with T2DM are obese or overweight ${ }^{4,5}$ and because several antidiabetic therapies cause weight gain. ${ }^{6}$ Excess weight contributes not only to the pathophysiology of diabetes, ${ }^{7}$ but also to an already elevated risk of cardiovascular disease in this population as an independent factor and through an increase in blood pressure and detrimental change in lipid profile. ${ }^{8}$ Consequently, recommendations for the prevention and treatment of diabetes have 
expanded beyond hyperglycemic control to also address multiple disease factors that affect the development and progression of the disease and/or diabetic comorbidities. ${ }^{9}$

There are challenges in treating T2DM with available therapeutic options. ${ }^{10}$ Most current therapies increase insulin levels or enhance insulin action. ${ }^{6}$ With a glucose-independent increase in insulin levels (either by direct injection of exogenous insulin or by increased secretion of endogenous insulin, as with sulfonylureas) comes a risk of hypoglycemia. This increase in insulin levels results in storage of excess glucose as fat. Additionally, as the disease advances and pancreatic insulin production declines, leading to subsequent loss of glycemic control, currently available agents require progressive dose escalation or the addition of other agents, which increases the complexity of the therapeutic regimen.

Sodium-glucose cotransporter-2 (SGLT2) inhibitors offer a novel mechanism for treatment of T2DM that is independent of insulin secretion or action. SGLT2 inhibitors have been shown in humans to: (1) promote weight loss, (2) be complementary to other, existing treatment modalities, and (3) have a low propensity to cause hypoglycemia. SGLT2 inhibitors are also expected to maintain effectiveness beyond the point of total pancreatic failure because the mechanism of action is not dependent on insulin. Beyond these advantages, SGLT2 inhibitors have the potential to modify the disease factors underlying the development and progression of diabetes. The weight loss seen with the SGLT2 inhibitors can, theoretically, partially restore insulin sensitivity. ${ }^{11}$

Experimental data in animals and humans show that both the beneficial and adverse effects of SGLT2 inhibition are consistent with the mechanism of action. These studies support a positive benefit-risk ratio for the treatment of T2DM with SGLT2 inhibition, and demonstrate that there is no apparent risk of kidney toxicity despite the site of drug action. The extensive body of work from the development of dapagliflozin, a potent, selective, and reversible SGLT2 inhibitor, validates the hypotheses generated from the mechanism of action and, moreover, indicates that treatment with dapagliflozin may play an important part in a comprehensive approach to the treatment of T2DM.

\section{SGLT-mediated glucose reabsorption}

Under normoglycemic conditions of $\sim 70-160 \mathrm{mg} / \mathrm{dL}$ plasma glucose, approximately $180 \mathrm{~g}$ of glucose pass through the glomeruli into the glomerular filtrate daily. ${ }^{12}$ Glucose is nearly completely reabsorbed via active transport as the filtrate passes through the proximal tubule on its way to the loop of Henle.

Within the proximal tubule, there are two families of glucose transporters (Figure 1A). ${ }^{13}$ SGLTs are located on the luminal surface of the proximal tubule and actively transport glucose against a concentration gradient by cotransport with $\mathrm{Na}^{+}$. On the basolateral surface, facilitative glucose transporters (GLUTs) passively transport glucose into the blood. Studies in rodents demonstrate that the majority of kidney glucose reabsorption is mediated by SGLT2 and GLUT2 in the S1 segment of the proximal tubule. ${ }^{14}$ The remaining glucose is reabsorbed in the distal straight segment of the proximal tubule by other GLUTs from these same protein families. ${ }^{12}$

Based on detection of mRNA, members of the SGLT family of transporters can be found in a variety of human tissues. SGLT1 has been found primarily in the small intestine, skeletal muscle, and heart, with lower expression detected in the trachea, brain, testis, kidney, and prostate; SGLT4 is expressed in the intestine, kidney, skeletal muscle, lung, and pancreas; SGLT5 has been found primarily in the kidney;

B

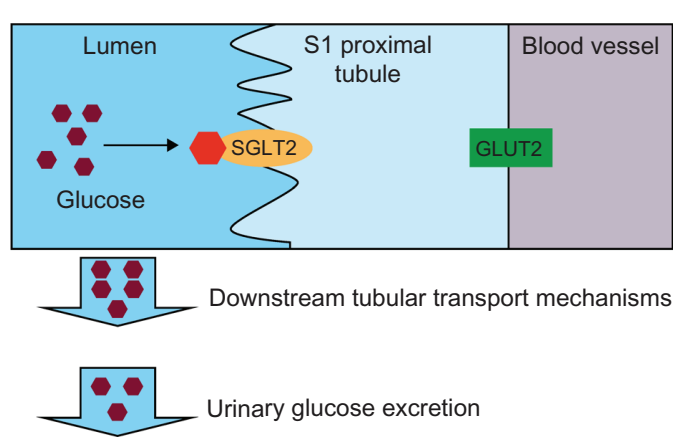

Figure I (A) In the normal range of blood glucose levels, the majority of glucose is reabsorbed in the kidney predominantly via the action of SGLT2 and GLUT2 in the SI segment of the proximal tubule. (B) SGLT2 inhibitors block the reabsorption of glucose in the SI proximal tubule under normal or hyperglycemic conditions, resulting in urinary glucose excretion.

Abbreviations: SGLT, sodium-glucose cotransporter; GLUT, glucose transporter. 
and SGLT6 is found in the brain, spinal cord, kidney, and small intestine. ${ }^{15-17}$ SGLT3, while a member of this family, is likely not a cotransporter but instead a glucose sensor found in the small intestine, skeletal muscle, testis, uterus, lung, brain, and thyroid. ${ }^{15,17}$ SGLT2 transporters have been found to be selectively expressed in the human kidney using several SGLT2-specific probes (Figure 2), and selectively expressed in rodent kidneys using an SGLT2-specific antibody. ${ }^{17,18}$ In instances where SGLT2 is not functional, such as inherited loss-of-function mutations in humans or genetic disruption in SGLT2 -/- mice, renal glucose reabsorption no longer occurs in the early proximal tubule. ${ }^{14,19}$ In keeping with the kidney-specificity of SGLT2 localization, in the rare instances of homozygous or compound heterozygous SGLT2 lossof-function mutations, patients are largely asymptomatic aside from profound glucosuria at normal levels of blood glucose while still maintaining normoglycemia. ${ }^{19}$ Given that profound glucosuria occurs in individuals bearing such mutations, other SGLT cotransporters do not fully compensate for loss of SGLT2 function. ${ }^{19}$

Control of renal glucose reabsorption appears to be independent of plasma insulin levels. Under hyperglycemic states, renal glucose reabsorption increases until it reaches a tubular glucose transport maximum..$^{20,21}$ Patients with
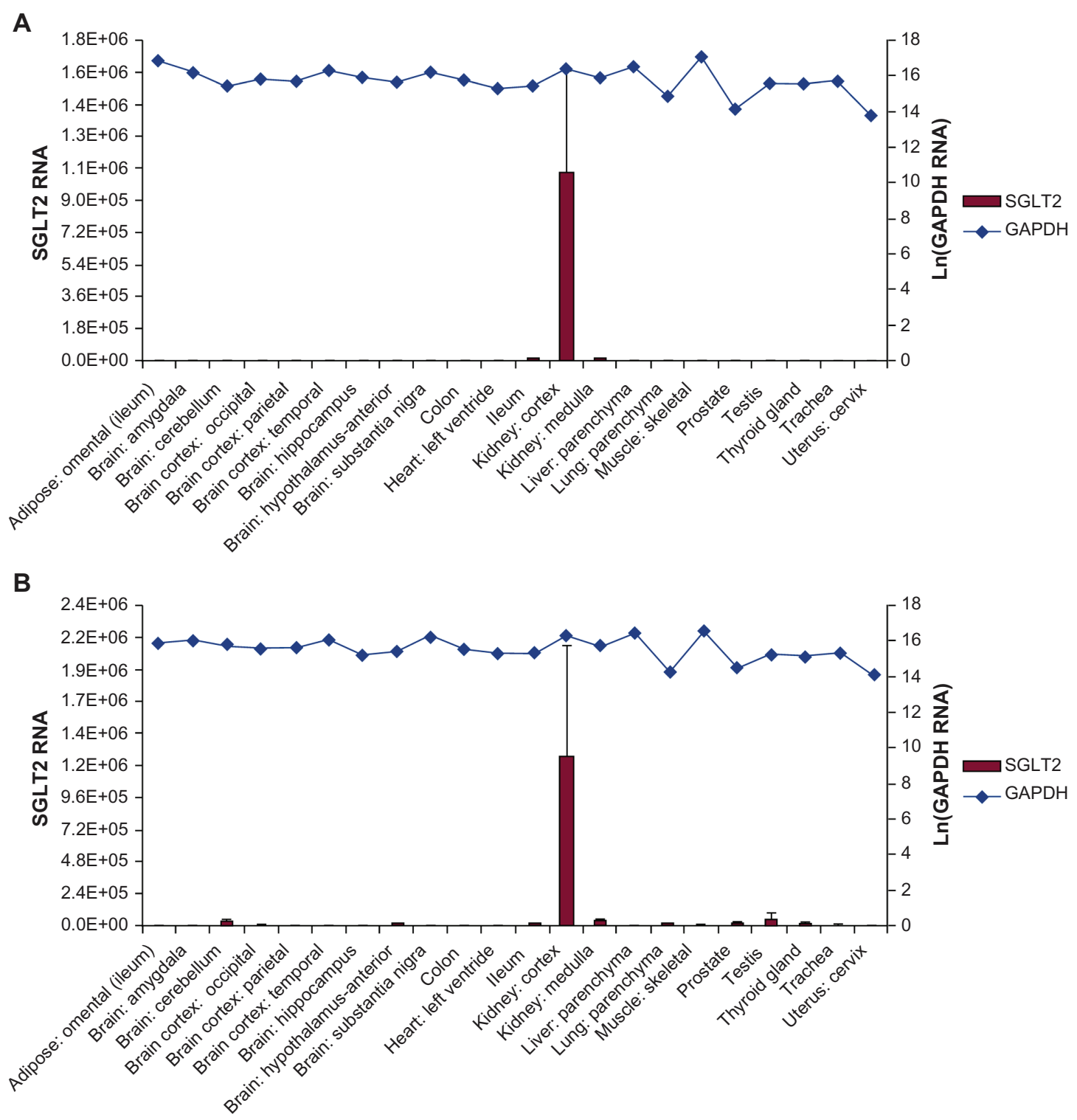

Figure 2 SGLT2 expression was determined in an array of 72 human tissues. Shown are representative tissues quantified using TaqMan quantitative PCR analysis with primer/ probe sets designed to amplify and detect regions of (A) exon 6-7 and (B) exon 13 of SGLT2 and normalized to GAPDH.

Note: Copyright (C) 2010. Reprinted with permission from Springer Science+Business Media B.V. Chen J, William S, Ho S, et al. Quantitative PCR tissue expression profiling of the human SGLT2 gene and related family members. Diabetes Ther. 2010;I(2):57-92. ${ }^{17}$

Abbreviations: SGLT, sodium-glucose cotransporter; PCR, polymerase chain reaction; GAPDH, glyceraldehyde-3-phosphate dehydrogenase. 
T2DM may have an increased tubular glucose transport maximum as a maladaptive response to sustained high glucose concentrations in the filtrate, contributing to already increased glycemic levels. ${ }^{22,23}$

\section{Phlorizin, a prototypical nonselective SGLT inhibitor}

The concept behind dapagliflozin and other SGLT inhibitors is that inhibition of renal glucose reabsorption and subsequent direct urinary excretion can reduce plasma glucose levels. Phlorizin, an SGLT inhibitor, was first isolated from the bark of apple trees in $1835 .{ }^{24}$ In the late 1800 s, phlorizin was demonstrated to induce urinary glucose excretion and was successfully used to lower the serum glucose of a patient with "diabetes." ${ }^{25,26}$ Despite the ability of phlorizin to lower serum glucose levels in humans, a clinical use has not been pursued.

Phlorizin has not been developed for clinical use for several reasons, including metabolic instability, low oral bioavailability, and a lack of selectivity for SGLT2. ${ }^{27}$ In addition to inhibiting SGLT2, phlorizin inhibits SGLT1, which is expressed in the intestine as well as the kidneys. ${ }^{15}$ In the intestine, SGLT1 is involved in glucose and galactose transport. Loss-of-function mutations in SGLT1 cause glucose-galactose malabsorption syndrome, resulting in severe diarrhea and dehydration on a glucose- or galactose-containing diet. ${ }^{13}$ Phlorizin is hydrolyzed to the compound phloretin, which also inhibits SGLT1 as well as multiple GLUT isoforms and results in impaired glucose transport. ${ }^{15,27}$

\section{Development of dapagliflozin}

Despite the limitations of phlorizin, interest in SGLT inhibition was renewed in the late 1980s, when Rossetti et al demonstrated that phlorizin-induced urinary glucose excretion reduced hyperglycemia in animals and normalized insulin sensitivity ${ }^{28}$ and $\beta$-cell function ${ }^{29}$ as a result of reduced glucotoxicity. The cloning of the SGLT transporters and identification of SGLT2 as the primary mediator of renal glucose reabsorption provided a new and specific target for development. ${ }^{19,30}$ The first class of SGLT2 inhibitors with O-glycoside linkages modeled after phlorizin were still susceptible to degradation in vivo. ${ }^{27,31}$ However, the next generation of SGLT2 inhibitors with C-glycoside linkages, the first of which was dapagliflozin, showed metabolic stability in vivo consistent with once-daily dosing, increased oral bioavailability, potency, and selectivity for SGLT2. ${ }^{27,32,33}$
Dapagliflozin is a reversible, highly specific inhibitor of SGLT2 and is the most advanced in clinical development of this class. In vitro studies have demonstrated that dapagliflozin has $>1400$-fold selectivity for SGLT2 relative to other SGLTs, SGLT1, SGLT4, and SGLT6, and to the related family member, SMIT. ${ }^{34}$ Dapagliflozin has at least 33,000-fold selectivity for SGLT2 over the facilitated glucose transporters GLUT1, GLUT2, and GLUT4, ${ }^{35}$ with the likely consequence that dapagliflozin will not interfere with basal or insulin-mediated glucose transport mediated by these transporters. Screening of $>300$ enzymes, transporters, ion channels, and receptors found no interactions with $10 \mu \mathrm{M}$ dapagliflozin, ${ }^{36}$ thus further reducing the potential for adverse effects based upon off-target receptor interactions.

\section{SGLT2 inhibition results in urinary glucose excretion \\ Animal models}

Like phlorizin, dapagliflozin was shown to induce urinary glucose excretion in animals (Figure 1B). In normal and diabetic rats (the Zucker Diabetic Fatty [ZDF] model), a single oral dose of dapagliflozin stimulated glucose excretion concomitantly with an increase in urine volume..$^{33}$ Onset of urinary glucose excretion in diabetic ZDF rats was apparent as early as 6 hours post-dose and continued for 24 hours (Figure 3). ${ }^{33}$ Diabetic rats excreted seven times as much glucose on a gram-per-animal basis than nondiabetic rats, demonstrating that the glucuretic action of dapagliflozin is proportional to the concentration of glucose in the blood.

Nondiabetic animals compensated for greater urine volume with increased water intake and compensated for the caloric loss by increasing food consumption. ${ }^{37}$ Despite increased food consumption, diet-induced obese rats treated with dapagliflozin experienced significant weight loss. A greater magnitude of weight loss was observed when the diet was controlled.

\section{Human studies}

In a 14-day study of dapagliflozin, patients with T2DM exhibited dose-dependent urinary glucose excretion of up to $70 \mathrm{~g}$ /day (approximately 280 calories)..$^{38}$ Glucose reabsorption was dose-dependently inhibited in the range of $20 \%-44 \%$ for doses of $5 \mathrm{mg}, 25 \mathrm{mg}$, and $100 \mathrm{mg}$ of dapagliflozin. ${ }^{38} \mathrm{~A}$ remaining degree of glucose reabsorption may be mediated by incomplete inhibition as higher concentrations of glucose compete with dapagliflozin for SGLT2 binding and/or by other tubular transport mechanisms in the distal end of the proximal tubule. 

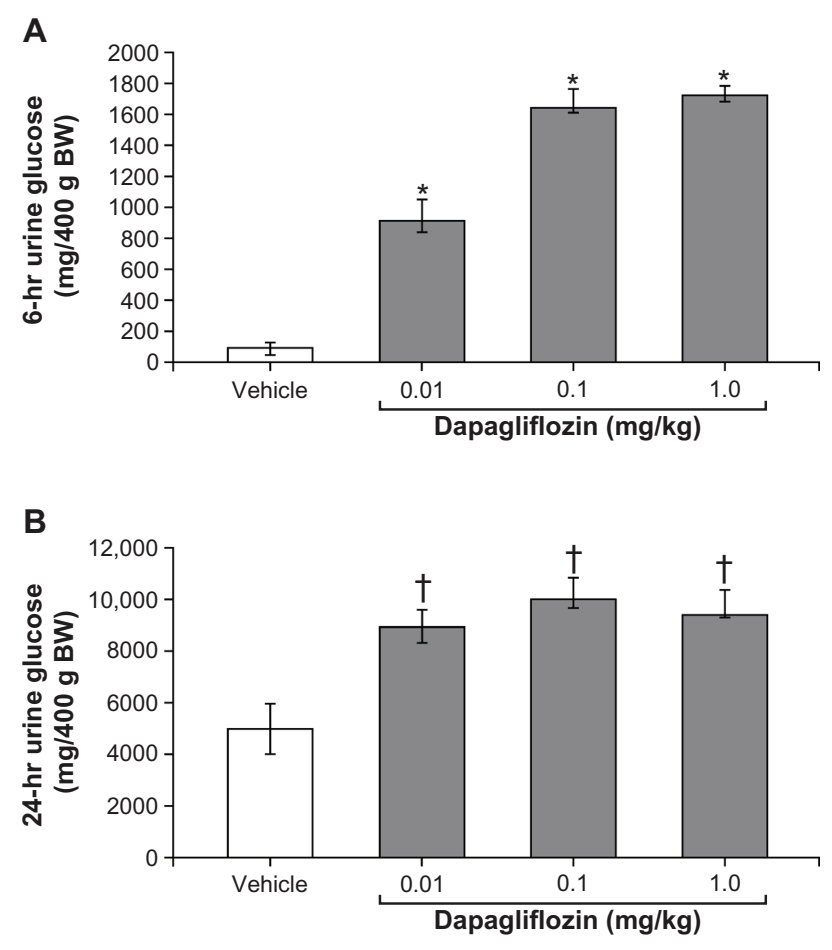

Figure 3 Single oral doses of dapagliflozin were demonstrated to stimulate an increase in urinary glucose excretion in Zucker diabetic fatty rats (A) over 6 hours, and (B) 24 hours post dose.

Notes: $* P<0.000$ I; ${ }^{\dagger} P<0.05$, each versus vehicle. Copyright (C) 2008. Reprinted with permission from American Diabetes Association. Han S, Hagan DL, Taylor JR, et al. Dapagliflozin, a selective SGLT2 inhibitor, improves glucose homeostasis in normal and diabetic rats. Diabetes. 2008;57(6): 1723-1729.33

Abbreviation: BW, body weight.

Dapagliflozin-induced urinary glucose excretion is proportional to the amount of glucose that is filtered through the glomeruli, ${ }^{39}$ which is a function of the plasma glucose concentration and the glomerular filtration rate (GFR). ${ }^{40}$ Consequently, in patients with reduced GFR (creatinine clearance $\leq 80 \mathrm{~mL} /$ minute) treated with dapagliflozin, the quantity of glucose in the urine is reduced compared with patients who have normal renal function (creatinine clearance $>80 \mathrm{~mL} /$ minute) ${ }^{41}$

\section{Urinary glucose excretion results in improved glycemic control Animal models}

Urinary glucose excretion in animal models translated into reduced hyperglycemia, as shown by decreased levels of blood glucose and hemoglobin A1c $\left(\mathrm{HbA}_{1 \mathrm{c}}\right)$. Over 2 weeks, treatment with dapagliflozin resulted in dose-dependent reductions of fasting plasma glucose (FPG) levels in ZDF rats to a mean of $138.2 \pm 7.4 \mathrm{mg} / \mathrm{dL}$ versus vehicle-treated rats at $295.2 \pm 19.5 \mathrm{mg} / \mathrm{dL}$ and, similarly, reductions in levels of plasma glucose in animals fed ad libitum (Figure 4). ${ }^{33}$

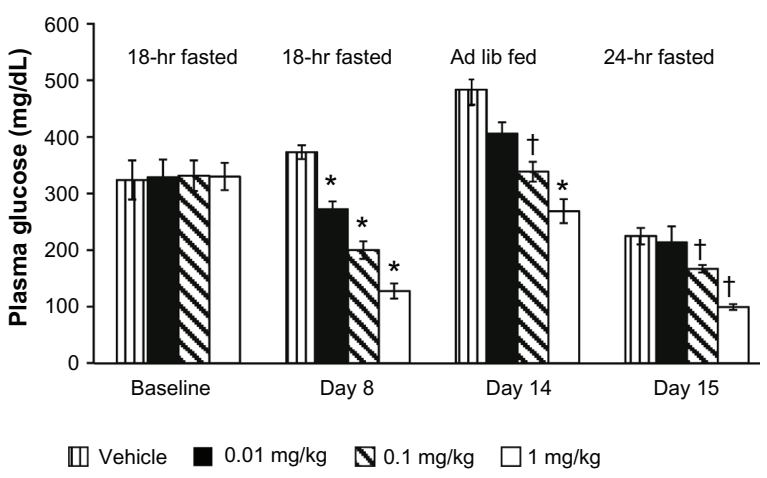

Figure 4 Once-daily oral administration of dapagliflozin resulted in significant, dose-dependent reductions in the plasma glucose of fed and fasting Zucker diabetic fatty rats over 15 days.

Notes: $* P<0.0001$; ${ }^{\dagger} P<0.05$, each versus vehicle. Copyright (C) 2008. Reprinted with permission from American Diabetes Association. Han S, Hagan DL, Taylor JR, et al. Dapagliflozin, a selective SGLT2 inhibitor, improves glucose homeostasis in normal and diabetic rats. Diabetes. 2008;57(6):1723-1729.33

Abbreviation: Ad lib, Ad libitum.

After 5 weeks of treatment, dapagliflozin maintained $\mathrm{HbA}_{1 \mathrm{c}}$ and nonfasting glucose in prediabetic, hyperglycemic, hyperinsulinemic, obese rats to levels near those observed in age-matched lean (nondiabetic) ZDF rats. ${ }^{42}$

\section{Human studies}

The potential therapeutic benefit suggested in animal models was confirmed in clinical studies. Phase III clinical trials showed glycemic efficacy in diverse patient populations, ranging from treatment naïve to those treated and with a long duration of diabetes (12 years or more) (Table 1). Treatment was found to be effective as monotherapy ${ }^{43,44}$ or as treatment combined with multiple diabetic treatment modalities (metformin, ${ }^{45}$ pioglitazone, ${ }^{46}$ glimepiride,${ }^{47}$ insulin ${ }^{48}$ ) for up to 2 years. ${ }^{49,50}$

As monotherapy ${ }^{43}$ or add-on to metformin,${ }^{45}$ dapagliflozin treatment resulted in significant placebo-subtracted decreases in $\mathrm{HbA}_{1 \mathrm{c}}$ of $-0.66 \%$ and $-0.54 \%$, respectively. In an exploratory monotherapy cohort, larger changes from baseline were seen in patients with an initial $\mathrm{HbA}_{1 \mathrm{c}} \geq 10.1 \% \cdot{ }^{43} \mathrm{HbA}_{1 \mathrm{c}}$ reductions were sustained for up to 2 years in an extension study conducted with dapagliflozin as add-on to metformin. Initial combination therapy with metformin plus dapagliflozin resulted in improvements in $\mathrm{HbA}_{1 \mathrm{c}}(-1.98 \%)$ that were significantly greater than with either metformin or dapagliflozin $(-1.44 \%$ and $-1.45 \%$, respectively) alone. ${ }^{44}$ Dapagliflozin was demonstrated to be noninferior to glipizide, a sulfonylurea, as an add-on to metformin; both resulted in a mean $\mathrm{HbA}_{1 \mathrm{c}}$ decrease of $-0.52 \%$ from baseline at 52 weeks. ${ }^{51}$

Similar results were observed when dapagliflozin was added on to insulin or agents that stimulate insulin secretion 


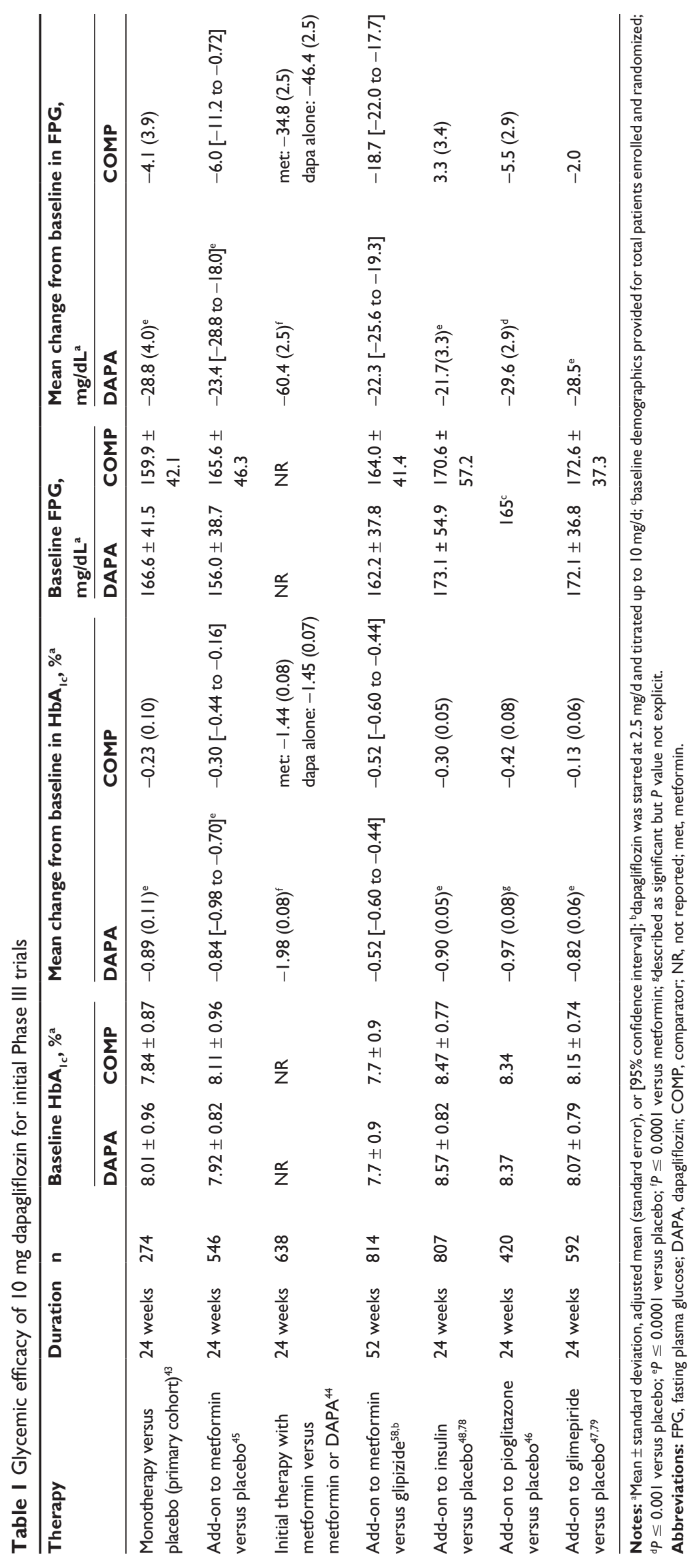


or enhance insulin action, namely, sulfonylureas and thiazolidinediones. As add-on to insulin ${ }^{48}$ or pioglitazone, ${ }^{46}$ dapagliflozin resulted in significant placebo-subtracted decreases in $\mathrm{HbA}_{1 \mathrm{c}}$ of $-0.60 \%$ and $-0.55 \%$, respectively, at 24 weeks that were sustained throughout 48 weeks. Dapagliflozin as add-on treatment to glimepiride resulted in a significant placebo-subtracted reduction in $\mathrm{HbA}_{1 \mathrm{c}}$ of $-0.68 \%$ over 24 weeks. ${ }^{47}$ With the exception of pioglitazone, the combination of dapagliflozin with these agents was associated with weight loss. ${ }^{47,48}$ In the case of pioglitazone, treatment with dapagliflozin reduced weight gain associated with pioglitazone treatment. ${ }^{46}$

FPG was significantly decreased in all studies. As monotherapy ${ }^{43}$ or add-on to metformin, ${ }^{45}$ dapagliflozin treatment resulted in significant placebo-subtracted reductions in FPG of $-24.7 \mathrm{mg} / \mathrm{dL}$ and $-17.5 \mathrm{mg} / \mathrm{dL}$, respectively, with the $10 \mathrm{mg}$ dose at week 24. Initial combination therapy with metformin plus dapagliflozin resulted in an improvement in FPG $(-60.4 \mathrm{mg} / \mathrm{dL})$ that was significantly greater than with either metformin or dapagliflozin (-34.8 $\mathrm{mg} / \mathrm{dL}$ and $-46.4 \mathrm{mg} / \mathrm{dL}$, respectively) alone. ${ }^{44} \mathrm{As}$ add-on to insulin ${ }^{48,78}$ or pioglitazone, ${ }^{46}$ dapagliflozin resulted in placebo-subtracted decreases in FPG of $-25.0 \mathrm{mg} / \mathrm{dL}$ and $-24.1 \mathrm{mg} / \mathrm{dL}$, respectively, at 24 weeks. Extension studies showed that reductions in FPG were sustained for up to 48 weeks with insulin ${ }^{48}$ or pioglitazone ${ }^{46}$ and up to 2 years with dapagliflozin in combination with metformin. ${ }^{49}$

Postprandial glucose (PPG) levels are an important aspect of overall glycemic control and have been shown to affect mortality risk independently of FPG levels. ${ }^{52}$ The effects of dapagliflozin on PPG were assessed in three different studies, ranging from 12-24 months. ${ }^{39,46,47}$ The $10 \mathrm{mg}$ dose of dapagliflozin decreased PPG levels in the range of -34.9 to $-71.5 \mathrm{mg} / \mathrm{dL}$ from baseline as monotherapy ${ }^{39}$ or in combination with glimepiride ${ }^{47}$ or pioglitazone. ${ }^{46}$ The magnitude of the decrease seemed to correspond to baseline PPG levels. Dapagliflozin as monotherapy resulted in a reduction of $-71.5 \mathrm{mg} / \mathrm{dL}$ from a baseline of $274.1 \mathrm{mg} / \mathrm{dL}$ after 12 weeks of treatment, ${ }^{53}$ and in combination with pioglitazone, dapagliflozin resulted in a decrease of $-67.5 \mathrm{mg} /$ $\mathrm{dL}$ from a baseline of $308.0 \mathrm{mg} / \mathrm{dL}$ after 24 weeks of treatment. ${ }^{46}$ In the trial evaluating dapagliflozin as add-on to glimepiride, dapagliflozin resulted in a decrease of $-60.6 \mathrm{mg} /$ $\mathrm{dL}$ from a baseline PPG level of $329.6 \mathrm{mg} / \mathrm{dL}$ (BristolMyers Squibb and AstraZeneca, data on file). The influence of baseline PPG levels and the PPG regulatory effects of dapagliflozin are most likely due to the proportional increases in glucose excretion as a result of increased filtered load.
Given the frequency of comorbid renal impairment in patients with T2DM, a study of dapagliflozin in patients with moderate renal impairment (estimated GFR $\geq 30$ and $\leq 59 \mathrm{~mL} / \mathrm{min} / 1.73 \mathrm{~m}^{2}$ ) was undertaken to assess the influence of GFR on dapagliflozin-induced urinary glucose excretion and clinical outcomes. ${ }^{54}$ An anticipated reduction in dapagliflozin efficacy was observed in these patients $\left(\mathrm{HbA}_{1 \mathrm{c}}\right.$, $-0.41 \%$ to $-0.44 \%$ vs placebo, $-0.32 \%$ ).

\section{SGLT2 inhibition has a low intrinsic potential for hypoglycemia Animal models}

The reduction in hyperglycemia with SGLT2 inhibition was demonstrated in animal and human studies to be associated with a low potential for hypoglycemia. There was no observed hypoglycemia in normoglycemic rats and dogs treated over the long term with supratherapeutic doses of dapagliflozin (at exposure multiples $>3000$ - and $>5500$-fold, respectively), suggesting that counter-regulatory responses were intact and also suggesting a low potential for hypoglycemia with selective SGLT2 inhibition (Bristol-Myers Squibb and AstraZeneca, data on file). Additionally, endogenous glucose production was mobilized in response to renal glucose loss in normal rats and in diabetic rats, with levels increasing in a dose-dependent manner, further supporting the preserved counter-regulatory response in both diabetic and nondiabetic animals. ${ }^{55}$ Consistent with this conclusion, hypoglycemia was not observed in nondiabetic or diabetic adult animals. ${ }^{33}$

\section{Human studies}

In the clinic, the risk of hypoglycemia is a limiting factor in the treatment of T2DM that restricts the ability to achieve good glycemic control, and is often seen with therapies that increase insulin secretion or with combination regimens that include such drugs. Dapagliflozin was expected to have a low propensity to cause hypoglycemia based on the selflimiting nature of renal glucose reabsorption, the preservation of counter-regulatory responses, the insulin-independent mechanism of action, and the selectivity of dapagliflozin for SGLT2 versus other renal glucose transporters. In clinical trials, dapagliflozin alone was associated with very few hypoglycemic episodes. These events were balanced between placebo and $10 \mathrm{mg}$ dapagliflozin given as monotherapy ${ }^{43}$ for 24 weeks (2.7\% and $2.9 \%$, respectively) or when dapagliflozin was given as add-on to metformin ${ }^{56}$ for 2 years $(5.8 \%$ and $5.2 \%$, respectively). When compared with glipizide, $10 \mathrm{mg}$ dapagliflozin as add-on to metformin was associated with 
a lower incidence of hypoglycemic events after 52 weeks (3.4\% dapagliflozin vs $39.7 \%$ glipizide). ${ }^{51}$ Like other antidiabetic drugs with low hypoglycemic potential, including glucagon-like peptide-1 receptor agonists, dipeptidyl peptidase-4 inhibitors, thiazolidinediones, and metformin, events of hypoglycemia were increased in combination with therapies with a known propensity to cause hypoglycemia. With $10 \mathrm{mg}$ dapagliflozin added on to either insulin for 48 weeks ${ }^{48}$ or glimepiride for 24 weeks, ${ }^{47}$ a higher proportion of patients experienced hypoglycemic events with dapagliflozin (insulin add-on, 53.6\%; glimepiride add-on, 7.9\%) versus placebo (insulin add-on, 51.8\%; glimepiride add-on, 4.8\%).

\section{Urinary glucose excretion results in weight loss}

\section{Animal models}

Preclinical studies of normoglycemic animals showed weight reductions in both rats and dogs. Similarly, urinary glucose excretion was shown to result in weight loss and/or reduced adiposity in hyperglycemic animals. Rats with diet-induced obesity showed a decrease in body weight of approximately $6 \%$ at the highest dose of dapagliflozin, despite increased consumption of food and water provided ad libitum. ${ }^{37}$ An even greater percentage of weight loss with dapagliflozin, $7 \%-12 \%$, was seen with diet restriction than in vehicletreated animals. Although some of the observed weight loss is attributable to water loss through diuresis, echo magnetic resonance imaging studies showed that dapagliflozin treatment resulted in decreased fat mass in these rats.

\section{Human studies}

As a result of the loss of 200-300 calories per day via glucose excretion in addition to diet and exercise counseling given to all patients in the clinical studies, a consistently favorable effect on body weight was observed with dapagliflozin treatment that manifested slightly differently in different treatment backgrounds. Weight loss was consistently seen across all Phase II and III studies, with the exception of add-on to pioglitazone at 48 weeks, where weight gain due to pioglitazone was mitigated (see below). ${ }^{46}$ For Phase III studies (24-52 weeks), weight loss ranged from $1.7 \mathrm{~kg}$ to $3.3 \mathrm{~kg}$ from baseline (Table 2). ${ }^{43-45,47,48,58}$ In patients with moderate renal impairment, dapagliflozin resulted in placebo-subtracted body weight reductions similar to those observed in other Phase III studies $(-2.4 \mathrm{~kg}$ to $-3.0 \mathrm{~kg}){ }^{54}$

As monotherapy, $10 \mathrm{mg}$ dapagliflozin resulted in weight loss ranging from $-2.7 \mathrm{~kg}$ to $-3.2 \mathrm{~kg}$ at 24 weeks; ${ }^{43,44}$ reductions were statistically different from those seen with metformin ${ }^{44}$ but not placebo. ${ }^{43}$ Added to weight-neutral metformin, weight loss was statistically different than with placebo ${ }^{45}$ and sustained through the study duration of 2 years $(-2.8 \mathrm{~kg}$ vs placebo, $-0.7 \mathrm{~kg}){ }^{49}$ Added to potentially weightincreasing oral antidiabetic drugs, dapagliflozin resulted in a significantly greater weight loss compared with glimepiride ${ }^{47}$ (dapagliflozin, $-2.26 \mathrm{~kg}$ vs placebo, $-0.72 \mathrm{~kg}$ ) and mitigated weight gain associated with pioglitazone ${ }^{46}$ up to week 48 (dapagliflozin, $+0.69 \mathrm{~kg}$ vs placebo, $+2.99 \mathrm{~kg}$ ). Escalating insulin doses are also associated with weight gain, in proportion to the reduction in glycemia. ${ }^{6}$ Added to ongoing insulin treatment, dapagliflozin maintained weight near baseline, while escalating insulin doses in the placebo group were associated with a steady weight gain through week 48 (dapagliflozin, $-1.5 \mathrm{~kg}$ vs placebo, $+0.9 \mathrm{~kg}$ ). ${ }^{48,57}$ In a study of patients uncontrolled on metformin alone, treatment with dapagliflozin resulted in increased fatty acid oxidation compared with placebo, supporting fat breakdown

Table 2 Body weight changes associated with $10 \mathrm{mg}$ dapagliflozin for initial Phase III trials

\begin{tabular}{|c|c|c|c|c|c|c|}
\hline \multirow[t]{2}{*}{ Therapy } & \multirow[t]{2}{*}{ Duration } & \multirow[t]{2}{*}{$\mathbf{n}$} & \multicolumn{2}{|c|}{ Baseline weight, $\mathbf{k g}^{\mathbf{a}}$} & \multicolumn{2}{|c|}{$\begin{array}{l}\text { Mean change from baseline } \\
\text { in weight, } \mathbf{k g}^{\mathrm{a}}\end{array}$} \\
\hline & & & DAPA & COMP & DAPA & COMP \\
\hline $\begin{array}{l}\text { Monotherapy versus placebo } \\
\text { (primary cohort) }^{43}\end{array}$ & 24 weeks & 274 & $94.2 \pm 18.7$ & $88.8 \pm 19.0$ & $-3.2(0.5)$ & $-2.2(0.4)$ \\
\hline Add-on to metformin versus placebo ${ }^{45}$ & 24 weeks & 546 & $86.3 \pm 17.5$ & $87.7 \pm 19.2$ & $-2.9[-3.3 \text { to }-2.4]^{\mathrm{e}}$ & $-0.9[-1.4$ to -0.4$]$ \\
\hline $\begin{array}{l}\text { Initial therapy with metformin versus } \\
\text { metformin or DAPA }{ }^{44}\end{array}$ & 24 weeks & 638 & NR & NR & $-3.33(0.24)^{f}$ & $\begin{array}{l}\text { met: }-1.36(0.24) \\
\text { dapa: }-2.73(0.23)\end{array}$ \\
\hline Add-on to metformin versus glipizide ${ }^{58, b}$ & 52 weeks & 814 & 88.4 & 87.6 & $-3.2[-3.6 \text { to }-2.9]^{g}$ & $\mathrm{I} .4[\mathrm{I} . \mathrm{I}$ to $\mathrm{I} .8]$ \\
\hline Add-on to insulin versus placebo ${ }^{48,78}$ & 24 weeks & 807 & $94.5 \pm 16.8$ & $94.5 \pm 19.8$ & $-1.67(0.18)^{\mathrm{e}}$ & $0.02(0.18)$ \\
\hline Add-on to pioglitazone versus placebo ${ }^{46}$ & 24 weeks & 420 & \multicolumn{2}{|c|}{$86 \mathrm{~kg}^{\mathrm{c}}$} & $-0.14(0.28)^{d}$ & $1.64(0.28)$ \\
\hline Add-on to glimepiride versus placebo ${ }^{47,79}$ & 24 weeks & 592 & $80.6 \pm 17.9$ & $80.9 \pm 15.8$ & $-2.26(0.22)^{\mathrm{e}}$ & $-0.72(0.23)$ \\
\hline
\end{tabular}

Notes: a Mean \pm standard deviation, adjusted mean (standard error), or [95\% confidence interval]; bdapagliflozin was started at $2.5 \mathrm{mg} / \mathrm{d}$ and titrated up to $10 \mathrm{mg} / \mathrm{d}$; 'baseline demographics provided for total patients enrolled and randomized; ${ }^{d} P \leq 0.00$ I versus placebo; ${ }^{\text {e }} P \leq 0.000$ I versus placebo; $P \leq 0.0$ I versus metformin; ${ }^{g} P \leq 0.000$ I versus glipizide.

Abbreviations: DAPA, dapagliflozin; COMP, comparator; NR, not reported; met, metformin. 
A

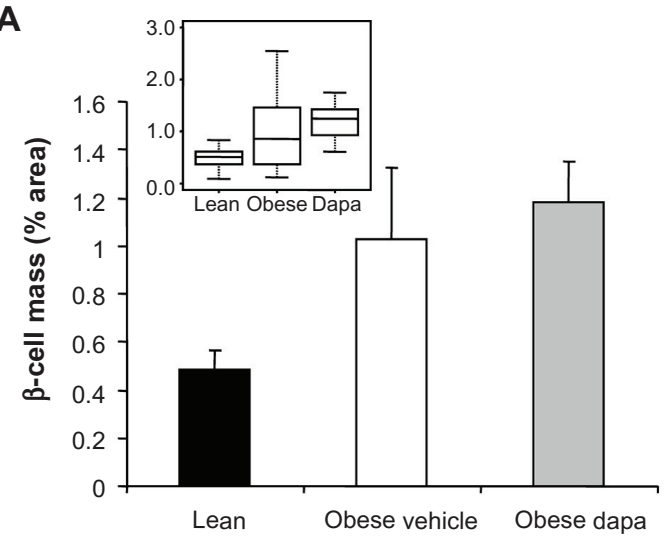

B

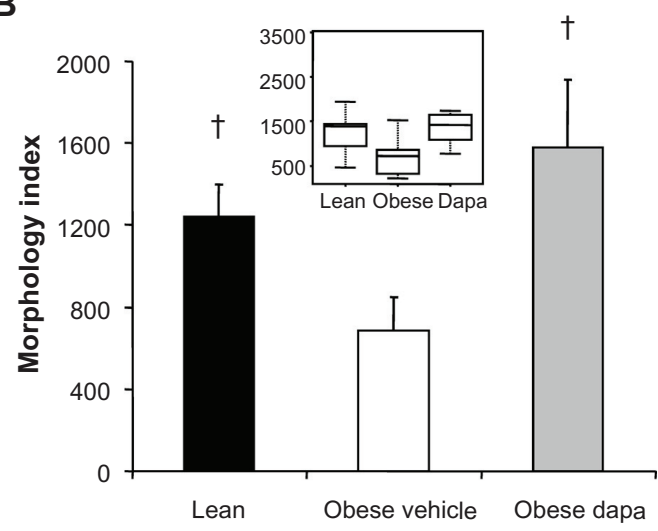

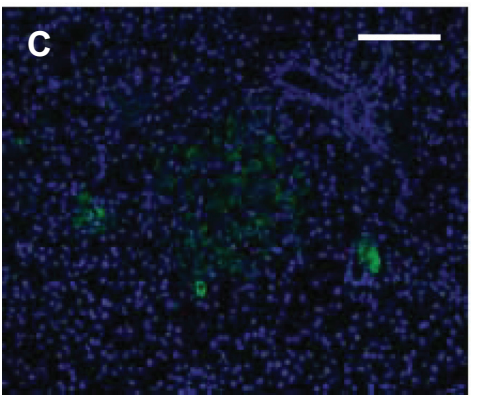
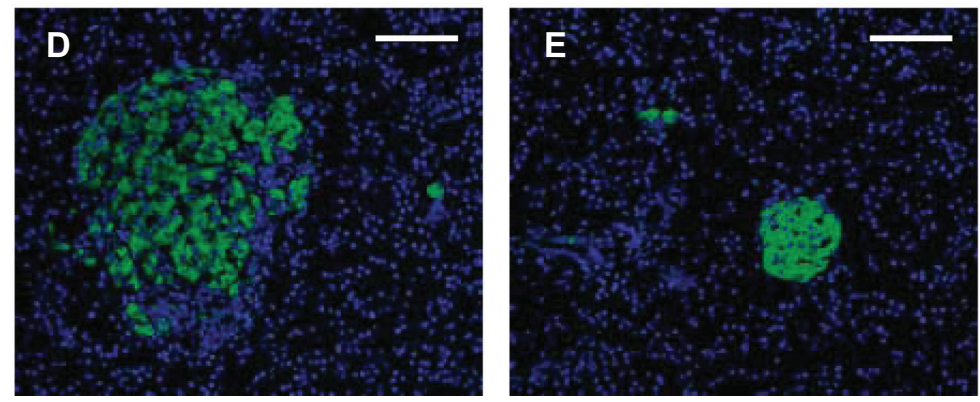

Figure 5 Dapagliflozin treatment of Zucker diabetic fatty rats at the initiation of a high-fat diet (A) did not have a significant effect on $\beta$-cell mass and (B) improved islet morphology. Box plots of the same data presented as insets. Representative 10x images of immunofluorescently stained $\beta$-cells (anti-insulin, green) and Hoescht-stained nuclei showed $(\mathbf{C})$ poor islet morphology and scattered $\beta$-cells in obese, vehicle-treated Zucker diabetic fatty rats compared with (D) improved islet morphology and insulin-staining intensity in dapagliflozin-treated animals. Islet size was increased in these obese, dapagliflozin-treated rats compared with (E) lean Zucker diabetic fatty rats.

Note: $t P<0.05$ versus obese fat-fed group. Copyright (c) 2010. Reprinted with permission from John Wiley and Sons. Macdonald FR, Peel JE, Jones HB, et al. The novel SGLT2 inhibitor dapagliflozin sustains pancreatic function and preserves islet morphology in obese, diabetic rats. Diabetes Obes Metab. 20I0;।2(II): I004-I0I2. ${ }^{60}$ Abbreviation: Dapa, dapagliflozin.

as the primary cause of weight loss. ${ }^{36,59}$ This finding is further supported by dual-energy X-ray absorptiometry data that showed a significantly larger decrease in adipose stores in patients treated with dapagliflozin in comparison with patients who received placebo. ${ }^{59}$

\section{Inhibition of SGLT2 ameliorates pathophysiology of T2DM}

$\beta$-cell dysfunction and insulin resistance underlie the development and progression of T2DM. ${ }^{60}$ SGLT2 inhibition is anticipated to improve both conditions. By reducing levels of blood glucose without stimulating insulin secretion, SGLT2 inhibition is anticipated to preserve $\beta$-cell function. Additionally, both glucose lowering and weight loss have been shown to increase insulin sensitivity. ${ }^{60}$

\section{Animal models}

In a study of female ZDF rats treated with dapagliflozin for 33 days at the initiation of a high-fat diet, dapagliflozin improved insulin sensitivity and resulted in improved islet morphology compared with vehicle-treated animals (Figure 5) ${ }^{61}$ Similar effects were observed in ZDF rats who were allowed to progress to moderate hyperglycemia before dapagliflozin intervention. Male, obese, prediabetic ZDF rats treated with dapagliflozin over the long term maintained plasma insulin levels compared with controls, in which plasma insulin levels declined, ${ }^{42}$ and despite unchanged glucose disposal and peripheral tissue uptake, endogenous glucose production was decreased, suggesting improved hepatic insulin sensitivity. ${ }^{33,42}$ Further, an insulin-sparing effect has been demonstrated in a knockout mouse model of SGLT2 ${ }^{62}$ Insulin exposure was 37\%-53\% less in SGLT2 knockout mice compared with control animals in response to an intraperitoneal glucose tolerance test.

\section{Human studies}

As suggested by the results of animal studies with dapagliflozin and in SGLT2 knockout mice, ${ }^{61,62}$ reduction in glucotoxicity due to decreased glucose levels improved $\beta$-cell function in a Phase III study of T2DM patients. ${ }^{63}$ T2DM patients receiving dapagliflozin as monotherapy or as add-on to metformin showed improvements in $\beta$-cell function as measured by Homeostasis Model Assessment 2 (HOMA-2 $\beta \%$ ). The placebo-corrected mean improvement in HOMA- $2 \beta \%$ 
across all dapagliflozin groups $(2.5 \mathrm{mg}, 5 \mathrm{mg}$, and $10 \mathrm{mg}$ doses) ranged from $13.2 \%$ to $17.3 \%$ for monotherapy and from $8.3 \%$ to $13.4 \%$ as add-on to metformin. However, the HOMA-2 $\beta \%$ model has not been fully validated in patients treated with SGLT2 inhibitors, and therefore, subsequent studies are required to confirm this effect. Results from a hyperinsulinemic euglycemic clamp study similarly showed an improved glucose disposal rate. ${ }^{64}$

\section{Side effects associated with urinary glucose excretion Electrolytes and vital signs}

\section{Animal models}

Consistent with the profile of a highly selective inhibitor, significant toxicity was not observed in rats with daily dosing of dapagliflozin at exposures up to 675-fold greater than human exposures in a 6-month study, or in dogs at exposures up to 3200-fold greater than human exposure in a 12-month study (Bristol-Myers Squibb and AstraZeneca, data on file). At exposures consistent with clinical doses, the side effects observed with dapagliflozin treatment in animals can be anticipated as a result of the mechanism of action.

Observed changes in urine composition were consistent with the osmotic diuretic effect of high urinary glucose levels. Osmotic diuretics are known to increase the urinary excretion of most electrolytes. ${ }^{65}$ In studies with dapagliflozin, there were no biologically relevant decreases in serum electrolytes in rats or dogs at supratherapeutic doses. In both rats and dogs, there were increases in the urinary excretion of calcium and phosphorus (Bristol-Myers Squibb and AstraZeneca, data on file). Normal mice treated with $10 \mathrm{mg} / \mathrm{kg}$ of dapagliflozin increased sodium excretion (Bristol-Myers Squibb and AstraZeneca, data on file). Increases in urinary sodium excretion were also observed in 1-month studies in rats and dogs but not in longer duration studies, such as the 6-month rat and 12-month dog toxicity studies, due to a presumed compensatory response.

\section{Human studies}

Similarly, in human studies, there was a transient increase in urinary sodium excretion. ${ }^{66}$ No changes were observed in mean serum sodium, calcium, or potassium levels. ${ }^{43,45,48,67}$ A small mean increase in serum magnesium $(0.30-1.0 \mathrm{mEq} / \mathrm{L})$ was seen in the $5 \mathrm{mg}$ groups of the monotherapy study. ${ }^{43}$ Similar small mean increases were observed in other clinical studies and are considered not likely to be of clinical relevance. ${ }^{48,58}$ Like other mild diuretics, $10 \mathrm{mg}$ dapagliflozin is associated across all studies to date with mean reductions in systolic $(-2.3$ to $-7.2 \mathrm{mmHg})$ and diastolic $(-1.0$ to $-2.8 \mathrm{mmHg}$ ) blood pressure, although this effect remains to be confirmed in studies controlling for concomitant antihypertensives. ${ }^{43,45,47,48,58,67}$ Orthostatic hypotension was rarely seen, although it has been reported in a healthy, normotensive subject in combination with a loop diuretic, bumetanide. ${ }^{68}$ Additionally, small mean increases in hematocrit and hemoglobin were observed in the first 12-16 weeks after starting dapagliflozin, with minimal increases thereafter; this was not associated with an increase in thromboembolic events. ${ }^{43,45,47,48,58,67}$

\section{Renal safety}

\section{Animal models}

Because of the renal mechanism of dapagliflozin, the kidney status/function was carefully evaluated in dapagliflozin-treated animals. Dapagliflozin administered for 2 weeks to rats with early streptozotocin-induced diabetes had no effect on kidney size or GFR. ${ }^{69}$ Supratherapeutic doses in rats with exposures up to 675 times human exposures at the expected maximum recommended dose showed no renal toxicity (Bristol-Myers Squibb and AstraZeneca, data on file). Histopathologic changes of rat renal tubular mineralization, minimal necrosis/ regeneration, and an exacerbation of chronic progressive nephropathy were not seen until very high exposure multiples ( $\geq 2116$-fold than maximum human exposure) in a 6-month study with daily dosing of dapagliflozin. Moreover, no renal histopathologic findings were observed in dogs despite exposure multiples $>3000$-fold. Mild proteinuria associated with the diuretic effect of dapagliflozin was observed in rats for exposures 85 -fold greater than the human exposures at the maximum recommended dose. Administration of diuretics in rats has previously been associated with increased urinary $\operatorname{protein}^{70}$ and $\mathrm{N}$-acetyl- $\beta$-D-glucosaminidase excretion ${ }^{71}$ in the absence of renal toxicity. Proteinuria was not observed in dogs except at very high exposures of greater than 3200-fold.

\section{Human studies}

Comparison of adverse events and laboratory data from a placebo-controlled pool of twelve Phase II and III clinical trials and from a dedicated study of moderate renal impairment show no reports of new or worsening renal impairment, progression of diabetic nephropathy, acute nephrotoxicity, such as acute tubular necrosis, or other events that would suggest toxic or immunologically mediated nephropathy. In the placebo-controlled pool, comprising largely normoalbuminuric patients, categorical 
shifts for urinary albumin:creatinine ratio were similar between dapagliflozin and placebo groups, with a similar proportion of patients remaining in their baseline category or transitioning to higher or lower categories (Bristol-Myers Squibb and AstraZeneca, data on file).

\section{Urinary tract infections (UTIs) and genital infections \\ Human studies}

Clinical studies were designed to monitor for signals of increased UTIs or genital infections, as it was hypothesized that the presence of glucose in the urine might provide a favorable growth environment for microorganisms. Signs, symptoms, and other events suggestive of UTIs and genital infections were increased in patients treated with dapagliflozin, were generally of mild or moderate intensity, and either resolved with self-treatment or responded readily to conventional interventions without the need to interrupt treatment. UTIs rarely resulted in treatment discontinuation (10 mg dapagliflozin, $0.3 \%$ vs placebo, $0.1 \%$ ). ${ }^{36}$ In a pooled analysis of twelve randomized Phase II and III studies (12-24 weeks), the set of adverse event terms was narrowed to exclude nonspecific symptoms such as dysuria or pruritus; diagnosed UTIs occurred in a slightly higher proportion of dapagliflozin patients (4.3\%) than controls (3.7\%). ${ }^{72}$ Events of pyelonephritis were reported infrequently and were balanced among subjects treated with dapagliflozin $(2.5 \mathrm{mg}$ : two cases; $5 \mathrm{mg}$ : one case; $10 \mathrm{mg}$ : zero cases) or control (one case). Genital infections, most often vulvovaginitis or balanitis, also occurred dose dependently in a higher proportion of dapagliflozin-treated patients $(4.8 \%)$ versus those who received placebo $(0.9 \%) .^{73}$

\section{Additional safety analyses and rare clinical events Neoplasms}

Animal models

Dapagliflozin was determined not to be genotoxic, and there were no dapagliflozin-related tumors in mice or rats at exposures over 100-fold greater than human exposures at the once-daily $10 \mathrm{mg}$ dose in humans (Bristol-Myers Squibb and AstraZeneca, data on file).

\section{Human studies}

Consistent with the preclinical safety data, there was no overall imbalance in neoplasms between dapagliflozin and control groups in the dapagliflozin clinical development program, with 4459 subjects exposed to dapagliflozin and 2239 subjects in the control group as of June 2010. An equal number of organ systems had an incidence rate ratio above and below unity. However, numeric imbalances in the small numbers of events were noted for breast and bladder cancer, with most events occurring during the first year of exposure. Long-term, large-scale surveillance of these rare events will be needed to rule out any association with dapagliflozin.

\section{Hepatic safety}

Animal models

Preclinical toxicology studies of mice, rats, and dogs found no histologic evidence of hepatotoxicity at exposures up to 5000 -fold greater than those observed at the $10 \mathrm{mg}$ dose in humans. ${ }^{36}$

\section{Human studies}

Clinical studies mirrored preclinical findings and showed no clinically meaningful differences in mean change from baseline for liver function tests between dapagliflozin and placebo groups. ${ }^{36}$ Signals of potential liver injury were assessed by an independent adjudication committee that was blinded to treatment. The small number of cases observed were balanced between dapagliflozin and control groups, and none were assessed as "definite", "highly likely" or "probably" related to treatment with dapagliflozin, though two cases were adjudicated as "probably" related to placebo. ${ }^{36}$ Severe drug-induced liver injury, defined as fatal or requiring liver transplantation, was not observed in any treatment group. There were two cases of elevated alanine aminotransferase or aspartate aminotransferase values $>3 \times$ the upper limit of normal and concomitant or subsequent total bilirubin $>2 \times$ the upper limit of normal that were assessed as "possible" in their relationship to dapagliflozin administration. In one case, the patient developed severe liver enzyme elevations and was diagnosed with drug-induced hepatitis and subsequently with probable autoimmune hepatitis after receiving dapagliflozin plus metformin.

\section{Bone safety}

Animal models

Trabecular bone accretion was increased in long-term toxicology studies of rats at exposures 2100-fold greater than human exposures with a $10 \mathrm{mg}$ dose, the maximum dose studied in Phase III clinical trials, but not in dogs at exposures greater than 3000-fold. ${ }^{36}$ Dapagliflozin is less selective for SGLT2 over SGLT1 in rats $(206 \times)$ compared with humans $(1400 \times)$. As a result, off-target inhibition of SGLT1 in rats could result 
in increased bacterial fermentation of intestinal glucose and subsequently increased calcium absorption in the gut. ${ }^{74-77}$

\section{Human studies}

In the overall clinical trial experience, there were no clinically meaningful changes in mean serum concentrations of calcium or in markers of bone formation and resorption, and there were no increases in events of fracture. ${ }^{36}$ Small mean increases were observed in levels of serum phosphorus, plasma magnesium, and parathyroid hormone that were not considered clinically relevant. A study of dapagliflozin treatment in patients with moderate renal impairment suggested that there is a potential risk of fracture in patients with stage $3 \mathrm{~b}$ moderate renal impairment (eGFR $\geq 30$ and $<45 \mathrm{~mL} / \mathrm{min} / 1.73 \mathrm{~m}^{2}$ ). As a result of the potential increased risk of fracture combined with reduced glycemic efficacy, dapagliflozin is not recommended for patients with an eGFR $<60 \mathrm{~mL} / \mathrm{min} / 1.73 \mathrm{~m}^{2}$.

\section{Conclusion}

The option of treating diabetes by inhibiting SGLT2 is a well-established historical idea now made possible by the development of a selective SGLT2 inhibitor. In addition to reducing blood glucose levels and hence glucose toxicity, SGLT2 inhibition directly disposes of calories in the form of excreted glucose. T2DM is believed to be a disease caused by caloric excess ${ }^{7}$ in the presence of genetic and other environmental factors, which have yet to be elucidated. SGLT2 inhibitors provide an important new mechanism with the potential to treat multiple aspects of the disease without a negative effect on body weight.

Most adverse events can be related back to dapagliflozin's glucuretic mechanism of action, with the exception of reported events of malignancy and a potentially drug-related case of hepatitis. Neither preclinical data nor mechanism of action supports a causal connection of dapagliflozin to these events. Nonetheless, these events merit further monitoring in ongoing trials to assess any potential safety signal. Although there are no clearly identified off-target adverse effects to date, the profile established in Phase III will need to be confirmed and refined with further experience in larger numbers of patients, as is the case for all diabetes medications. The expected adverse events, namely, increased UTIs and genital infections, were generally mild and effectively treated with standard of care in clinical studies. Most importantly, results from a comprehensive nonclinical toxicology program and a diverse set of monotherapy and add-on clinical trials demonstrated a reassuring renal safety profile in the general T2DM population studied.
Dapagliflozin provides an alternative therapy that may be used alone or in combination with other antihyperglycemic therapies; to date, the drug has demonstrated efficacy, a favorable safety profile, and a low potential for hypoglycemia. Because the mechanism of action is insulin independent, dapagliflozin offers a truly novel option for the treatment of T2DM and for better control of concomitant risk factors and potentially diabetic complications. Development continues to further define the benefit-risk profile.

\section{Acknowledgments}

Funding support was provided by Bristol-Myers Squibb and AstraZeneca. Editorial support was provided by William Watkins and Alexandra Silveira of PAREXEL.

\section{Disclosure}

Jean Whaley, Mark Tirmenstein, Timothy Reilly, and James List are currently employees of and/or stockholders in BristolMyers Squibb. Simon Poucher, JoAnne Saye, and Shamik Parikh are currently employees of and/or stockholders in AstraZeneca.

\section{References}

1. Holman RR, Paul SK, Bethel MA, Matthews DR, Neil HA. 10-year follow-up of intensive glucose control in type 2 diabetes. $N$ Engl J Med. 2008;359(15):1577-1589.

2. Nathan DM, Cleary PA, Backlund JY, et al; Diabetes Control and Complications Trial/Epidemiology of Diabetes Interventions and Complications (DCCT/EDIC) Study Research Group. Intensive diabetes treatment and cardiovascular disease in patients with type 1 diabetes. $N$ Engl J Med. 2005;353(25):2643-2653.

3. Gaede P, Vedel P, Larsen N, Jensen GV, Parving HH, Pedersen O. Multifactorial intervention and cardiovascular disease in patients with type 2 diabetes. N Engl J Med. 2003;348(5):383-393.

4. Cheung BM, Ong KL, Cherny SS, Sham PC, Tso AW, Lam KS. Diabetes prevalence and therapeutic target achievement in the United States, 1999 to 2006. Am J Med. 2009;122(5):443-453.

5. Eeg-Olofsson K, Cederholm J, Nilsson PM, et al. Risk of cardiovascular disease and mortality in overweight and obese patients with type 2 diabetes: an observational study in 13,087 patients. Diabetologia. 2009;52(1): 65-73.

6. Nathan DM, Buse JB, Davidson MB, et al. Medical management of hyperglycemia in type 2 diabetes: a consensus algorithm for the initiation and adjustment of therapy: a consensus statement of the American Diabetes Association and the European Association for the Study of Diabetes. Diabetes Care. 2009;32(1):193-203.

7. Bays H, Mandarino L, DeFronzo RA. Role of the adipocyte, free fatty acids, and ectopic fat in pathogenesis of type 2 diabetes mellitus: peroxisomal proliferator-activated receptor agonists provide a rational therapeutic approach. J Clin Endocrinol Metab. 2004;89(2):463-478.

8. Anderson JW, Konz EC. Obesity and disease management: effects of weight loss on comorbid conditions. Obes Res. 2001;9 Suppl 4: 326S-334S.

9. Handelsman Y, Mechanick JI, Blonde L, et al. American Association of Clinical Endocrinologists Medical Guidelines for Clinical Practice for developing a diabetes mellitus comprehensive care plan. Endocr Pract. 2011;17 Supp1 2:S1-S53. 
10. Cryer PE. Hypoglycaemia: the limiting factor in the glycaemic management of type I and type II diabetes. Diabetologia. 2002;45(7): 937-948.

11. Bays H, Blonde L, Rosenson R. Adiposopathy: how do diet, exercise and weight loss drug therapies improve metabolic disease in overweight patients? Expert Rev Cardiovasc Ther. 2006;4(6):871-895.

12. Marsenic O. Glucose control by the kidney: an emerging target in diabetes. Am J Kidney Dis. 2009;53(5):875-883.

13. Wright EM, Hirayama BA, Loo DF. Active sugar transport in health and disease. $J$ Intern Med. 2007;261(1):32-43.

14. Vallon V, Platt KA, Cunard R, et al. SGLT2 mediates glucose reabsorption in the early proximal tubule. JAm Soc Nephrol. 2011;22(1): 104-112.

15. Wright EM, Loo DD, Hirayama BA. Biology of human sodium glucose transporters. Physiol Rev. 2011;91(2):733-794.

16. Grempler R, Augustin R, Froehner S, et al. Functional characterisation of human SGLT-5 as a novel kidney-specific sodium-dependent sugar transporter. FEBS Lett. 2011;586(3):248-253.

17. Chen J, William S, Ho S, et al. Quantitative PCR tissue expression profiling of the human SGLT2 gene and related family members. Diabetes Ther. 2010;1(2):57-92.

18. Sabolic I, Vrhovac I, Balen ED, et al. Expression of Na+-D-glucose cotransporter SGLT2 in rodents is kidney-specific and exhibits sex and species differences. Am J Physiol Cell Physiol. 2012. [Epub ahead of print.]

19. Santer R, Calado J. Familial renal glucosuria and SGLT2: from a mendelian trait to a therapeutic target. Clin J Am Soc Nephrol. 2010;5(1): 133-141.

20. Abdul-Ghani MA, DeFronzo RA. Inhibition of renal glucose reabsorption: a novel strategy for achieving glucose control in type 2 diabetes mellitus. Endocr Pract. 2008;14(6):782-790.

21. Bays H. From victim to ally: the kidney as an emerging target for the treatment of diabetes mellitus. Curr Med Res Opin. 2009;25(3): 671-681.

22. Mogensen CE. Maximum tubular reabsorption capacity for glucose and renal hemodynamics during rapid hypertonic glucose infusion in normal and diabetic subjects. Scand J Clin Lab Invest. 1971;28(1):101-109.

23. List JF, Whaley JM. Glucose dynamics and mechanistic implications of SGLT2 inhibitors in animals and humans. Kidney Int Suppl. 2011; 120:S20-S27.

24. Petersen C. Analyse des Phloridzins. Annales Académie Science Française. 1835;15:178. French.

25. Von Mering J. Ueber kunstlichen diabetes. Centralbl Med Wiss. 1886; 22:531. German.

26. Achard C, Delamare V. The bark of the apple root, phlorizin, reduces diabetic hyperglycemia. Soc Medic Des Hopitaux. 1899:379-393.

27. Chao EC, Henry RR. SGLT2 inhibition - a novel strategy for diabetes treatment. Nat Rev Drug Discov. 2010;9(7):551-559.

28. Rossetti L, Smith D, Shulman GI, Papachristou D, DeFronzo RA. Correction of hyperglycemia with phlorizin normalizes tissue sensitivity to insulin in diabetic rats. J Clin Invest. 1987;79(5): 1510-1515.

29. Rossetti L, Shulman GI, Zawalich W, DeFronzo RA. Effect of chronic hyperglycemia on in vivo insulin secretion in partially pancreatectomized rats. J Clin Invest. 1987;80(4):1037-1044.

30. Wells RG, Pajor AM, Kanai Y, Turk E, Wright EM, Hediger MA. Cloning of a human kidney cDNA with similarity to the sodium-glucose cotransporter. Am J Physiol. 1992;263(3 Pt 2):F459-F465.

31. Washburn WN. Evolution of sodium glucose co-transporter 2 inhibitors as anti-diabetic agents. Expert Opin Ther Pat. 2009;19(11):1485-1499.

32. Obermeier M, Yao M, Khanna A, et al. In vitro characterization and pharmacokinetics of dapagliflozin (BMS-512148), a potent sodiumglucose cotransporter type II inhibitor, in animals and humans. Drug Metab Dispos. 2010;38(3):405-414.

33. Han S, Hagan DL, Taylor JR, et al. Dapagliflozin, a selective SGLT2 inhibitor, improves glucose homeostasis in normal and diabetic rats. Diabetes. 2008;57(6):1723-1729.
34. Uveges A, Hagan D, Onorato J, et al. Dapagliflozin selectively inhibits human SGLT2 versus SGLT1, SMIT1, SGLT4, and SGLT6. Presented at the American Diabetes Association 71st Scientific Sessions; June 24-28, 2011. San Diego, CA: 2011:Poster P-987.

35. Poucher SM, DeSchoolmeester J, Ehenborn J, Vernon W, Clementz T. Dapagliflozin selectivity for glucose transporters GLUT1, 2, and 4. Presented at the American Diabetes Association 71st Scientific Sessions; June 24-28, 2011. San Diego, CA: Poster P-1041.

36. Bristol Myers-Squibb, AstraZeneca. US Food and Drug Administration Endocrinologic and Metabolic Advisory Committee background document: dapagliflozin, BMS-512148, NDA 202293. US Food and Drug Administration. Available from: www.fda. gov/AdvisoryCommittees/CommitteesMeetingMaterials/Drugs/ EndocrinologicandMetabolicDrugsAdvisoryCommittee/ucm262993. htm. Accessed July 20, 2011.

37. Devenny J, Harvey S, Rooney S, et al. The effect of dapagliflozin, a highly selective SGLT-2 inhibitor on body weight in diet-induced obese rats. Obesity. 2007;15(Suppl 19):A121.

38. Komoroski B, Vachharajani N, Feng Y, Li L, Kornhauser D, Pfister M. Dapagliflozin, a novel, selective SGLT2 inhibitor, improved glycemic control over 2 weeks in patients with type 2 diabetes mellitus. Clin Pharmacol Ther. 2009;85(5):513-519.

39. Salsali A, Hruba V, Ying L, et al. Reduction in postprandial glucose with dapagliflozin in type 2 diabetes [abstract]. Diabetes. 2011; 60:A286:1104-P

40. Gerich JE. Physiology of glucose homeostasis. Diabetes Obes Metab. 2000;2(6):345-350.

41. Kasichayanula S, Liu X, Pe Benito M, LaCreta F, Boulton DW. Influence of renal function on dapagliflozin pharmacodynamics in patients with type 2 diabetes mellitus [abstract]. Presented at the American Society of Nephrology; November 8-13, 2011. Philadephia, PA: 2011:TH-PO525.

42. Zinker B, Ma X, Liu H, et al. Chronic dapagliflozin treatment reduces elevated hepatic glucose production and enhances pancreatic insulin content in male ZDF rats [abstract]. Diabetes. 2011;60:A283: 1031-P.

43. Ferrannini E, Ramos SJ, Salsali A, Tang W, List JF. Dapagliflozin monotherapy in type 2 diabetic patients with inadequate glycemic control by diet and exercise: a randomized, double-blind, placebo-controlled, phase III trial. Diabetes Care. 2010;33:2217-2224.

44. Henry R, Murray A, Marmolejo MH, Hennicken D, Ptaszynska A, List JF. Dapagliflozin, metformin-XR, or both together as initial therapy for T2DM [abstract]. Diabetes. 2011;60:A84:307-OR.

45. Bailey CJ, Gross JL, Pieters A, Bastien A, List JF. Effect of dapagliflozin in patients with type 2 diabetes who have inadequate glycaemic control with metformin: a randomised, double-blind, placebo-controlled trial Lancet. 2010;375(9733):2223-2233.

46. Rosenstock J, Vico M, Wei L, Salsali A, List J. Dapagliflozin addedon to pioglitazone reduces $\mathrm{HbAl} \mathrm{c}$ and mitigates weight gain with low incidence of hypoglycemia in type 2 diabetes [abstract]. Diabetes. 2011; 60:A270:986-P.

47. Strojek K, Yoon KH, Hruba B, Elze M, Langkilde AM. Effect of dapagliflozin in patients with type 2 diabetes who have inadequate glycaemic control with glimepiride: a randomized, 24-week, double-blind, placebo-controlled trial. Diabetes Obes Metab. 2011; 13(10):928-938.

48. Wilding JP, Woo V, Soler NG, et al. Long-term efficacy of dapagliflozin in patients with type 2 diabetes mellitus receiving high doses of insulin: a randomized trial. Ann Intern Med. 2012;156(6):405-415.

49. Bailey CJ, Gross JL, Yadav M, Iqbal N, Mansfield TA, List JF. Long-term efficacy of dapagliflozin as add-on to metformin (MET) in T2DM inadequately controlled with MET alone [abstract]. Diabetes. 2011;60: A271:988-P.

50. Woo V, Tang W, Salsali A, List JF. Long-term efficacy of dapagliflozin monotherapy in patients with type 2 diabetes mellitus [abstract]. Presented at the International Diabetes Federation World Diabetes Congress; December 4-8, 2011. Dubai, United Arab Emirates: 2011:D-0991. 
51. Nauck M, Del Prato S, Rohwedder K, Theuerkauf A, Langkilde A, Parikh S. Long-term efficacy and safety of add-on dapagliflozin vs add-on glipizide in patients with T2DM inadequately controlled with metformin: 2-year results. Presented at the American Diabetes Association 71st Scientific Sessions; June 24-28, 2011. San Diego, CA: 2011:40-LB.

52. Bavenholm PN, Efendic S. Postprandial hyperglycaemia and vascular damage - the benefits of acarbose. Diab Vasc Dis Res. 2006;3(2): 72-79.

53. List JF, Woo V, Morales E, Tang W, Fiedorek FT. Sodium-glucose cotransport inhibition with dapagliflozin in type 2 diabetes. Diabetes Care. 2009;32(4):650-657.

54. Kohan DE, Fioretto P, List J, Tang W. Efficacy and safety of dapagliflozin in patients with type 2 diabetes and moderate renal impairment [abstract]. J Am Soc Nephrol. 2011;Suppl:22:TH-PO524.

55. Zinker B, Ma X, Liu H, Washburn W, Whaley J. Acute glucose fluxes following a single dose of dapagliflozin [abstract]. Diabetes. 2011;60: A273:995-P.

56. Bailey CJ, Gross JL, Hennicken D, Iqbal N, Mansfield TA, List JF. Long-term efficacy of dapagliflozin as add-on to metformin in T2DM inadequately controlled with metformin alone. Presented at the American Diabetes Association 71st Scientific Sessions; June 24-28, 2011. San Diego, CA: 2011:988-P.

57. Wilding JPH, Woo V, Soler NG, Pahor A, Sugg J, Parikh S. Sustained effectiveness of dapagliflozin over 48 weeks in patients with type 2 diabetes poorly controlled with insulin [abstract]. Diabetes. 2010;59:LB7. Abstract 21-LB.

58. Nauck MA, Del Prato S, Meier JJ, et al. Dapagliflozin versus glipizide as add-on therapy in patients with type 2 diabetes who have inadequate glycemic control with metformin: a randomized, 52-week, double-blind, activecontrolled noninferiority trial. Diabetes Care. 2011;34(9): 2015-2022.

59. Bolinder J, Ljunggren O, Kullberg J, et al. Effects of dapagliflozin on body weight, total fat mass and regional adipose tissue distribution in patients with type 2 diabetes mellitus with inadequate glycemic control on metformin. J Clin Endocrinol Metab. 2012. [Epub ahead of print.]

60. Kahn SE. The relative contributions of insulin resistance and beta-cell dysfunction to the pathophysiology of type 2 diabetes. Diabetologia. 2003;46(1):3-19.

61. Macdonald FR, Peel JE, Jones HB, et al. The novel SGLT2 inhibitor dapagliflozin sustains pancreatic function and preserves islet morphology in obese, diabetic rats. Diabetes Obes Metab. 2010;12(11): 1004-1012.

62. Jurczak MJ, Lee HY, Birkenfeld AL, et al. SGLT2 deletion improves glucose homeostasis and preserves pancreatic beta-cell function. Diabetes. 2011;60(3):890-898.

63. Salsali A, Bastien A, Mansfield T, Ying L, Ravichandran S, List JF. Dapagliflozin improves hyperglycemia and beta-cell function without increasing hypoglycemic episodes in patients with type 2 diabetes mellitus [abstract]. Presented at the American Association of Clinical Endocrinologists; April 13-17, 2011. San Diego, CA: 2011:204.

64. Mudaliar S, Henry R, Boden G, et al. Changes in insulin sensitivity as measured by glucose disposal rate and acute insulin secretion with the sodium glucose co-transporter 2 inhibitor dapagliflozin. Presented at the 47th Annual Meeting of the European Association for the Study of Diabetes; September 12-16, 2011. Lisbon, Portugal: 2011: Abstract 854 .
65. Lang F. Osmotic diuresis. Ren Physiol. 1987;10(3-4):160-173.

66. Heerspink HJ, List J, Boulton D, Liu X, Ying L, deZeeuw D. The SGLT2 inhibitor dapagliflozin, a proximal tubular diuretic with antihypertensive properties? Presented at the World Congress of Nephrology 2011; April 8-12, 2011. Vancouver, Canada: 2011:Abstract SU183.

67. Wilding JP, Norwood P, T'joen C, Bastien A, List JF, Fiedorek FT. A study of dapagliflozin in patients with type 2 diabetes receiving high doses of insulin plus insulin sensitizers: applicability of a novel insulin-independent treatment. Diabetes Care. 2009;32(9):1656-1662.

68. Wilcox CS, Liu X, Kasichayanula S, et al. Evaluation of interactions of dapagliflozin and bumetanide. Presented at the American Society of Nephrology; November 16-21, 2010. Denver, CO: 2010.

69. Thomson SC, Miracle C, Rieg T, Whaley J, Vallon V. Chronic SGLT2 blockade reduces proximal reabsorption and normalizes state of tubuloglomerular feedback activation in hyperfiltering diabetic rats [abstract]. J Am Soc Nephrol. 2009;20:734A:2723.

70. Kutina AV, Zakharov VV, Natochin YV. Excretion of proteins by rat kidney during various types of diuresis. Bull Exp Biol Med. 2008; 146(6):671-674.

71. Lina BA, Bos-Kuijpers MH, Til HP, Bar A. Chronic toxicity and carcinogenicity study of erythritol in rats. Regul Toxicol Pharmacol. 1996;24(2 Pt 2):S264-S279.

72. Parikh S, Johnsson K, Ptaszynska A, Schmitz B, Sugg J, List JF. Characterization of urinary tract infections in the setting of pharmacologically induced glucosuria [abstract]. Diabetes. 2011;60: A270:984-P

73. List JF, Ley S, Ptaszynska A, et al. Characterization of genital infections in the setting of pharmacologically-induced glucosuria [abstract]. Diabetes. 2011;60:A270:985-P.

74. Campbell JM, Fahey GC Jr, Wolf BW. Selected indigestible oligosaccharides affect large bowel mass, cecal and fecal short-chain fatty acids, $\mathrm{pH}$ and microflora in rats. $J$ Nutr. 1997;127(1):130-136.

75. Kasahara M, Maeda M, Hayashi S, Mori Y, Abe T. A missense mutation in the $\mathrm{Na}(+) /$ glucose cotransporter gene SGLT1 in a patient with congenital glucose-galactose malabsorption: normal trafficking but inactivation of the mutant protein. Biochim Biophys Acta. 2001; 1536(2-3):141-147.

76. Wright EM. Genetic disorders of membrane transport. I. Gluocse galactose malabsorption. Gastrointest Liver Physiol. 1998;275(5): G879-G882.

77. Zafar TA, Weaver CM, Zhao Y, Martin BR, Wastney ME. Nondigestible oligosaccharides increase calcium absorption and suppress bone resorption in ovariectomized rats. J Nutr. 2004;134(2):399-402.

78. Wilding JPH, Woo V, Soler NG, et al. Dapagliflozin in patients with type 2 diabetes poorly controlled on insulin therapy - efficacy of a novel insulin-independent treatment [abstract]. Diabetes. 2010;59: A21-A22:78-OR.

79. Strojek K, Hruba V, Elze M, et al. Efficacy and safety of dapagliflozin in patients with type 2 diabetes mellitus and inadequate glycaemic control on glimepiride monotherapy. Diabetologia. 2010;53:S347:870-P.

\section{Publish your work in this journal}

Diabetes, Metabolic Syndrome and Obesity: Targets and Therapy is an international, peer-reviewed open-access journal committed to the rapid publication of the latest laboratory and clinical findings in the fields of diabetes, metabolic syndrome and obesity research. Original research, review, case reports, hypothesis formation, expert opinion and commentaries are all considered for publication. The manuscript management system is completely online and includes a very quick and fair peer-review system, which is all easy to use. Visit http://www.dovepress.com/testimonials.php to read real quotes from published authors. 